\title{
Ultrasonic Identification of Weld Defects Made by Butt Welding with Hot Plate on Plastic Pi- pelines
}

Martin Faturík, Miloš Mičian, Radoslav Koňár

University of Žilina, Univerzitná 1, Department of Mechanical Engineering Technologies; E-mail: martin.faturik@fstroj.uniza.sk, milos.mician@fstroj.uniza.sk, radoslav.konar@fstroj.uniza.sk

This article describes usage of ultrasonic method TOFD for testing of welds on plastic pipelines welded with butt fusion with hot plate. As an artificial defects were selected cold fusion and inclusion in the weld area, by using metallic tape and wire as a mentioned defects. To test defects was necessary to use wedges with water as a bonding agent. Results of testing are proof of usefulness of this method. It can severely reduce risks of accidents caused by unidentified defects, which can occur in welded joints. Since plastic pipelines are used even in nuclear industry as a replacement for metal pipelines for its resistance against radiaton decay, it is necessary to implement similar testing regulations to avoid fatal incidents and method like TOFD and Phased Array ultrasonic testing can prevent such failures.

Keywords: ultrasonic defectoscopy, TOFD, plastic pipelines, gas industry

\section{Acknowledgement}

Article was created within project solution VEGA: 1/0836/13, KEGA: 034ZU-4/2015 a KEGA: 014ZU-4/2015.

\section{References}

[1] KOVÁČIK, M., HYŽA, R. (2013). Ultrazvuková skúška časti obvodového zvaru čpavkovej tlakovej nádoby technikami Phased Array a TOFD. [Online] 2013. [Date: 21. 04 2013.] http://www.ssndt.sk/files/odborne/PA\%20a\%20TOFD\%20na\%20cpavku.pdf.

[2] KOVÁČIK, M. (2010). Skúšanie materiálov ultrazvukom. Bratislava : s.n., 2010.

[3] MARTANČÍK, B. (2012). Výskum diagnostiky defektov pomocou nových ultrazvukových metód TOFD a Phased Array a vplyv na životnost’ zváraných konštrukcií. Bratislava : Slovenská technická unverzita v Bratislave, 2012. s. 151, dizertačná práca.

[4] OBRAZ, J. (1989). Zkoušení materiálu ultrazvukem. Praha : SNTL Nakladatelství technické literatury, 1989. s. 464. ISBN 80-03-00097-1.

[5] OLYMPUS. (2004). Introduction to Phased Array Ultrasonic Technology Applications. Waltham, MA : Olympus NDT, 2004. s. 351. ISBN 0-9735933-0-X.

[6] OLYMPUS. (2012). Phased Array Testing Basic Theory for Industrial Applications. 2nd ed. Waltham, MA : Olympus NDT, 2012. s. 113. DMTA-20003-01EN.

[7] LOYDA, M., ŠPONER, V., ONDRÁČEK, L a kol. (2001). Svařování termoplasti̊. Praha. Uno, 2001. ISBN 80223-6603-6

[8] MORAVEC, J., BRADÁČ, J., BERAN, D., NOVÁKOVÁ, I. (2014). The Impact of Thermal Cycles of Superheated Steam on Pipes Material of By-Pass of Steam and Gas-Steam. In: 23rd International Conference on Metallurgy and Materials, Metal 2014, Brno, 2014. ISBN 978-80-87294-52-9.

[9] SEJČ, P. BIELAK, R. - ŠVEC, P. - ROŠKO, M. (2006). Computer simulation of heat affected zone during MIG brazing of zinc-coated steel sheets. In Kovové materiály. Metallic materials. Roč. 44, č. 4 (2006), s.225-234. ISSN 0023-432X.

[10]MEŠKO, J. - Zrak, A. - MULCZYK, K. - SZYMON, T. (2014). Microstructure analysis of welded joints after laser welding. In: Manufacturing technology : journal for science, research and production. - ISSN 1213-2489. Vol. 14, no. 3 (2014), s. 355-359.

[11]NORBERT, R. - MEŠKO, J. - ZRAK, A. (2014). Technology of laser forming. In: Manufacturing technology: journal for science, research and production. - ISSN 1213-2489. - Vol. 14, no. 3 (2014), s. 428-431. 